\title{
A New Differential Privacy Crowdsensing Scheme Based on the Multilevel Interactive Game
}

\author{
Sungwook Kim $\mathbb{D}$ \\ Department of Computer Science, Sogang University, 35 Baekbeom-ro (Sinsu-dong), Mapo-gu, Seoul, 04107, Republic of Korea
}

Correspondence should be addressed to Sungwook Kim; swkim01@sogang.ac.kr

Received 16 May 2018; Revised 20 September 2018; Accepted 3 October 2018; Published 17 October 2018

Academic Editor: Jun Cai

Copyright (C) 2018 Sungwook Kim. This is an open access article distributed under the Creative Commons Attribution License, which permits unrestricted use, distribution, and reproduction in any medium, provided the original work is properly cited.

With the rapid growth of network of devices with embedded technology, mobile crowdsensing (MCS) has been gaining increasing popularity. The development of $5 \mathrm{G}$ network services is prompting further growth in crowdsensing applications. However, MCS participants risk their privacy when reporting data with their actual sensing positions. To address this issue, the concept of differential privacy (DP) can be adopted to provide a theoretical guarantee for participants' privacy in MCS services. In this study, we design a new DP crowdsensing scheme with game theory. Based on the multilevel interactive game model, MCS server, DP controllers, and mobile devices are regarded as rational individual decision makers that aim to maximize their own payoffs. For these decision makers, the proposed game approach analyzes suitably the competitive and coordinative MCS environments. The main novelty possessed by our control scheme is to capture the dynamics of MCS system operations with the privacy consideration. Compared with other existing protocols, performance evaluation shows the advantages of our proposed scheme in terms of the sensing task success ratio, MCS participating ratio, and normalized payoff of participating devices. Finally, we provide the guidance on the future research direction of MCS services including other issues.

\section{Introduction}

A large number of network devices are being connected at an unprecedented rate realizing the idea of the Internet of Things (IoT). The interconnections of these devices enable us to collect and exchange data for offering various applications, such as product tracking, environment monitoring, patient surveillance, and home automation. It opens the door to innovations that facilitate new interactions among 'things' and human and provides new opportunities for applications and services that improve the quality of our daily life. Cisco predicts that 50 billion devices would connect to the Internet by 2020; this number would reach 500 billion by 2025 . In the future, IoT will lead to a significant change in our life patterns in the way we live and interact with multiple devices [1-4].

For the IoT implementation, mobile crowdsensing (MCS) is an appealing paradigm and has gained growing attention in the last years. MCS refers to the technology that uses mobile network devices to sense and collect the information of surrounding environments and then report this information to the MCS server. With built-in data storage and intelligent functional ability, the MCS server analyzes statistical characteristics and mines the data to reveal hidden attributes based on the mass of collected information. Taking advantage of the ubiquitous presence of powerful mobile devices, that is, smartphones or sensor-embedded actuators, MCS has become an appealing technique to commercial businesses that wish to collect data without making largescale investments. In the recent years, famous companies use the MCS technique to offer services based on the big data collected [5]

The performance of MCS depends on the contribution of participating devices. However, mobile devices acting MCS services have to sacrifice their energy and time. To stimulate cooperative actions among selfish devices, the MCS server should pay the price for each participating device. It can induce devices to provide full sensing efforts while ensuring the sensing quality. More specifically, each individual device is motivated to transmit accurate sensing information, if the expected payment from the MCS server exceeds its sensing cost [6]. Otherwise, it has nothing to gain by reporting the MCS information. Therefore, adaptive pricing policy is a key 
challenge in the MCS system to balance the profits between the MCS server and individual devices.

As a mathematical tool, game theory has been used to investigate the interaction between the MCS server and mobile devices. Game theory is the study of models of conflict and cooperation between intelligent rational decision makers [7]. In this paper, we develop a new game model to formulate the operation of MCS system. Our proposed game model is understood to achieve greater and reciprocal advantages for selfish network agents, who can strengthen their competitive advantages by using the multilevel interactive approach. In addition, to get a desired game outcome, learning algorithms are used in competitive environments. During the iterative learning process, three key steps are performed by game players: (i) observing the current system environment, (ii) estimating the prospective payoff, and (iii) selecting a best strategy to reach an effective solution.

Traditionally, people value their privacy and they certainly do not want their personal information to be accessible to just anyone at any time. But recent advances in technology threaten privacy and open up the possibility of a range of negative consequences as a result of access to personal data. During MCS operations, the collected information can be sensitive to individuals, revealing personal data such as individual commuting routes and locations. Therefore, ensuring the privacy and security of personal information collected through MCS is important and necessary. Therefore, privacy protection mechanism has been considered an essential requirement for the MCS system. However, until now, the principal privacy issue in the MCS system has not yet been well addressed; few existing work systematically investigates the privacy protection problem considering the tradeoff between privacy preservation and sensed data accuracy [811].

In 2006, C. Dwork et al. introduced the concept of differential privacy (DP), which is a mathematical definition for the privacy loss that results to individuals when their private information is used in the creation of a data product. The main idea of DP is that a network agent perturbs its original data by adding carefully designed random noises and then directly transmits the noisy data to a data collection server. Finally, the data collector is able to compute population statistics. The DP approach can limit the increased privacy risk resulting from inclusion of private data in a statistical database and makes it possible to provide very accurate statistics from the database while still ensuring high levels of privacy protection. Therefore, DP ensures that the privacy of data is protected because the data collector cannot access the original data $[12,13]$. In real-world environments, DPbased data collection is first implemented in Google Chrome browser to collect and track the client-side information [14].

Motivated by the above discussion, we have adopted the DP mechanism to design a new practical MCS control scheme. For dealing with the DP-based MCS situation, our scheme focuses on the three control issues: MCS price decision, privacy level selection, and MCS contribution handling. In the MCS price decision process, the MCS price is decided by a novel learning algorithm. In the privacy level selection process, mobile devices dynamically select their privacy levels to maximize their profits. In the MCS contribution handling process, the sensed data amount of each device is adjusted using another online learning algorithm. Based on the interactive feedback mechanism, control decisions in each process are made in a distributed manner and cause cascade interactions while balancing the payoff among selfish system agents. Finally, we can reach the most profitable solution in the MCS system.

1.1. Related Work. Considerable state-of-the-art research has been conducted on the design of DP-based MCS schemes. Reference [15] investigates the influence of sensing data correlation on DP protection and explores different correlation models to describe the relationship from two different perspectives. From a protector's view, Bayesian network is used to model the probabilistic relationship among sensing data, and a corresponding DP is given. From an adversary's view, the Gaussian correlation model is used to describe the data correlation structure, and a new algorithm to compute Bayesian DP leakage is presented. Finally, the extensive simulations show the influence of the size of maximum correlated group in all of participants on Bayesian DP [15].

Jian Lin and others designed the Privacy-Preserving Mobile Crowdsensing (PPMC) scheme to achieve approximate computational efficiency, individual rationality, and social cost minimization [16]. First, authors consider a singlebid model in which each user can only submit a set of tasks and then develop another multibid model in which each user can submit a bid for each task in its task set. One important component of both models is a platformdefined score function for selecting users. In particular, two score functions, that is, linear and log functions, are developed to realize the two models. Extensive numerical results demonstrate that the PPMC scheme can achieve bidprivacy preservation though sacrificing social cost [16].

Reference [17] proposes a new DP aggregation protocol to resist collusion attacks without incurring extra error. This protocol ensures that the accumulated error in the sum statistics is only a copy Laplace noise required for DP, but the magnitude of the noise incorporated to each party' data is not large enough to the data privacy. Another feature of the proposed protocol is that it needs not to have a priori estimation of those colluded parties to make it secure under insecure communication channels. In addition, authors design an efficient aggregation encryption scheme to support MCS applications and also make some extensions to make the proposed protocol more applicable in realities, such as the fault tolerant and supporting parties' dynamic joins or leaves [17].

Reference [18] proposes a new anonymized datacollection scheme that can estimate data distributions more accurately. In particular, authors propose two methods: Single to Randomized Multiple Dummies (S2M) and S2M with Bayes ( $\mathrm{S} 2 \mathrm{Mb})$, both of which can make a better tradeoff between privacy and utility. And then, they develop an algorithm that calculates optimized values of the parameters that constitute $\mathrm{S} 2 \mathrm{M}$ and $\mathrm{S} 2 \mathrm{Mb}$. The optimized parameters satisfy DP and minimize the expected values of mean squared errors and Jensen-Shannon divergence, which are 
the popular utility metrics. Finally, this study provides the implementation results of synthetic and real datasets and proves that $\mathrm{S} 2 \mathrm{M}$ and $\mathrm{S} 2 \mathrm{Mb}$ methods outperform existing schemes [18].

Leye Wang and others designed the Sparse Differential Location Privacy (SDLP) scheme to provide a theoretical guarantee for participants' location privacy regardless of an adversary's prior knowledge [19]. For the privacy preserving, this scheme includes three functions: data adjustment, optimal location obfuscation, and uncertainty aware inference functions. The data adjustment function is used to fit the original sensing data to the obfuscated location. The optimal obfuscation function minimizes the uncertainty in data adjustment under the constraints of DP and evenly distributed obfuscation. The uncertainty aware inference function improves the inference accuracy for the obfuscated data. The SDLP scheme explores how to balance three key functions for space MCS applications. Empirical evaluation with real-world datasets shows that the SDLP scheme can provide adequate privacy protection with reduced data quality loss [19].

Some earlier studies [15-19] have attracted considerable attention while introducing unique challenges in handling the MCS control problems. In this paper, we demonstrate that our proposed scheme significantly outperforms these existing PPMC [16] and SDLP [19] schemes.

1.2. Contribution. For the MCS process, there is little work that has perfectly resolved the tradeoff between efficiency and privacy. In order to strike the appropriate performance balance among contradictory requirements, we take a first step towards addressing the DP-based MCS control problem by presenting a multilevel game model. Our game process is operated according to the step-by-step repeated game manner while harnessing the synergies among MCS agent interactions. Based on the novel multilevel game approach, the proposed scheme achieves effective advantages to adapt to dynamically changing MCS environments. Although several MCS algorithms including the DP concept have been proposed, no systematic study including the strategic control issues has been conducted.

1.3. Organization. The remainder of this article is organized as follows. In the next section, we describe the principal platform of the MCS system and present our multilevel game formulation. And then, we explain the proposed MCS control algorithms in detail and their properties. Section III provides the simulation scenario and the experimental results. By means of a comparison with existing PPMC [16] and SDLP [19] schemes, we validate the performance excellency of the proposed scheme. Finally, we present our conclusion and discuss the remaining open challenges in this research area along with possible solutions.

\section{The Proposed MCS Control Algorithms}

In this section, we first give the formation of multilevel game model and notations of important parameters. After that we present the proposed MCS control algorithms with necessary assumptions. Finally, the proposed scheme is described strategically in the nine-step procedures.

2.1. MCS System Architecture and Multilevel Game Model. In this study, we consider the MCS infrastructure with the DP-management platform. It is consisting of mobile devices, MCS server, and DP controllers. The MCS server gets sensing data and pays the price through the DP controllers. There are multiple sensing tasks requesting from the MCS server, which delivers a final service to MCS customers by analyzing the obtained information. As MCS participants, mobile devices sense independently the information data while deciding their level of privacy protection and the amount of MCS contributions. When a mobile device chooses a higher level of privacy protection or smaller amount of sensed data, its service payment is reduced; the service payment of each individual mobile device is proportional to his contribution. Therefore, each rational mobile device needs to tradeoff between his own preference and the payoff maximization.

DP controllers control the exchange of sensed data between multiple devices and the MCS server. According to the level of privacy protection, there are multiple DP controllers. Each DP controller has been assigned as a specific privacy level and works as a mediator to collect the sensed data with the same DP level. With their selected privacy levels, mobile devices report the sensed data to the corresponding DP controller. According to their contributions, the MCS server pays the service price to DP controllers and each individual DP controller redistributes the allocated price to its corresponding mobile devices. The general platform of hierarchical MCS system is shown in Figure 1.

DP is a privacy definition that was originally developed by Dwork, Nissim, McSherry, and Smith, with major contributions by many others over the years. It is one of the most important privacy metrics and has been widely studied in data-mining research publications. In cryptography, the DP aims to provide the accuracy of queries from statistical databases while minimizing the chances of identifying its records. Simply, the DP concept is satisfied if the distribution of the output does not change observably when one mobile device's information in the dataset is changed [18]. Specifically, a common mechanism adds the random noise generated from a Laplace distribution to the query result. It must guarantee that the contribution of each individual device's information to a query result is limited according to the $\varepsilon$ parameter, which is a positive real number $[14,20]$.

Originally, DP was designed for the data sharing scenario in which a trusted data server holds a dataset where each tuple is collected from a device without perturbation and releases perturbed information from the dataset using a randomized algorithm [20]. However, this traditional DP approach is not adaptable to implement the DP-based MCS service. If the DP controller is untrusted, then each individual device would have to perturb his tuple to protect privacy before it is collected by the DP controller. In this case, the tuple owned by each mobile device is regarded as a singleton dataset, and we require that the perturbation algorithm $\mathfrak{I}$ should locally ensure the DP concept when such a singleton dataset is 


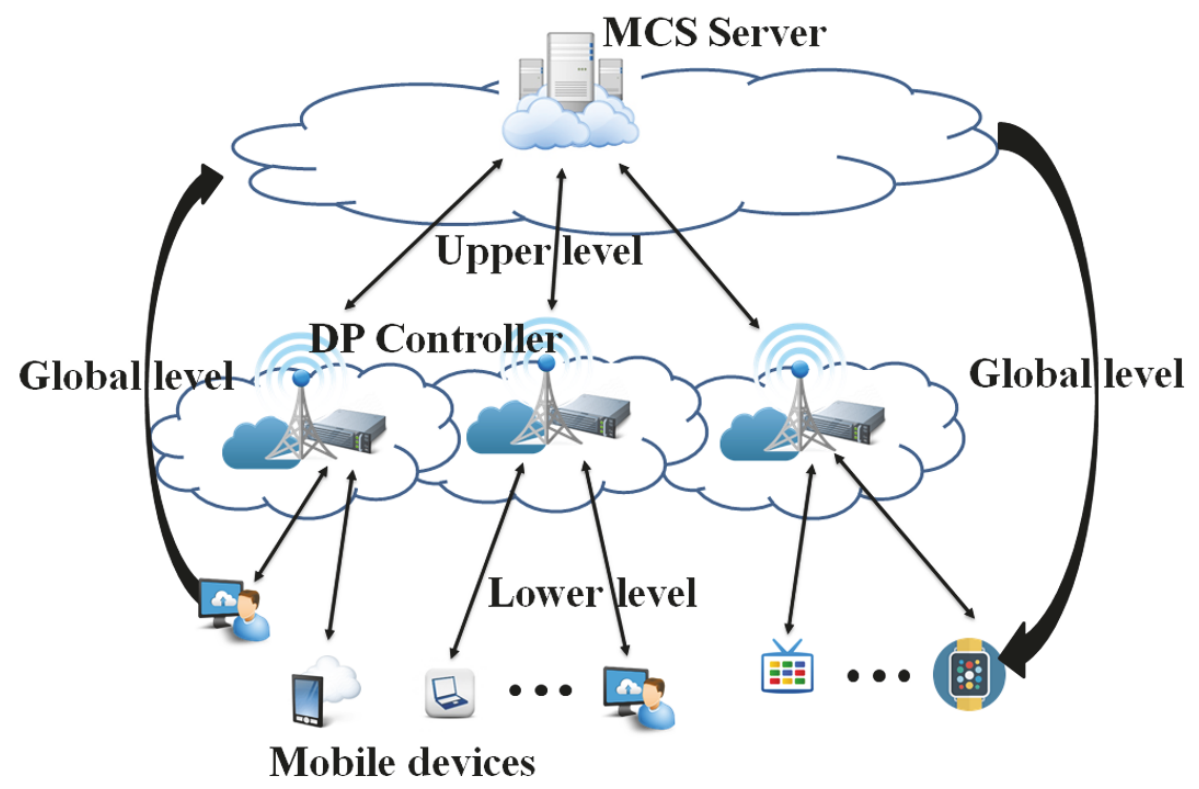

FIGURE 1: The general architecture of a multilevel MSC system platform.

given as the input. This variant of DP is referred to as $\varepsilon$-local differential privacy $(\varepsilon$-LDP) $[14,20]$. In this study, we focus on the $\varepsilon$-LDP instead of the original notion of $\varepsilon$-DP; the $\varepsilon$ LDP approach provides a much stronger degree of privacy protection for each mobile device against the DP controller.

During the MCS system operations, system agents, that is, MCS server, DP controllers, and mobile devices, make decisions individually. In this situation, a main issue for each agent is how to perform well by considering the mutualinteraction relationship. To develop a new dynamic MCS control scheme, we design a new multilevel game model to balance conflict of interests among system agents. At the upper level, the MCS server pays the price to multiple DP controllers by using a proportional-split sharing manner. At the lower level, DP controllers and mobile devices are game players. Each DP controller redistributes the obtained price to mobile devices, and mobile devices dynamically select their corresponding DP controllers to maximize their payoffs. At the global level, the MCS server and mobile devices are game players; they interact with each other to get a wellbalanced MCS system performance. At each level, game players dynamically adjust their decisions to maximize their own profits. For the implementation practicality, our multilevel game model $(\mathbb{G})$ is designed in an entirely distributed and self-organizing interactive fashion. Mathematically, $\mathbb{G}$ can be defined as $\mathbb{G}=\{\mathbb{N}=\{\mathcal{S}, \mathscr{C}, \mathscr{D}\}, \mathbb{A}, \mathbb{E}, \mathbb{S}=$ $\left.\left\{\boldsymbol{S}^{\mathcal{S}},\left\{\boldsymbol{S}_{C_{i} \in \mathscr{C}}^{\mathscr{C}}\right\},\left\{\boldsymbol{S}_{D_{i} \in \mathscr{D}}^{\mathscr{D}}\right\}\right\}, \mathbb{U}=\left\{U^{\mathcal{S}},\left\{U_{C_{i} \in \mathscr{C}}^{\mathscr{C}}\right\},\left\{U_{D_{j} \in \mathscr{D}}^{\mathscr{D}}\right\}\right\}, T\right\}$ at each time period $t$ of gameplay.

(i) $\mathbb{N}=\{\mathcal{S}, \mathscr{C}, \mathscr{D}\}$ is the finite set of game players where $\mathcal{S}$ represents one MCS server, $\mathscr{C}=\left\{C_{1}, \ldots, C_{l}\right\}$ is a set of multiple DP controllers, and $\mathscr{D}=\left\{D_{1}, \ldots, D_{n}\right\}$ is a set of MCS participating mobile devices.

(ii) $\mathbb{A}=\left\{A_{1} \cdots A_{v}\right\}$ is the set of MCS sensing application tasks. (iii) $\mathbb{E}=\left\{\varepsilon_{C_{1}} \cdots \varepsilon_{C_{l}}\right\}$ is the discrete DP levels of MCS; $\varepsilon_{C_{1 \leq i \leq l}}$ in $\mathbb{E}$ represents the DP level of DP controller $C_{i}$.

(iv) $\mathbb{S}=\left\{\boldsymbol{S}^{\mathcal{S}},\left\{\boldsymbol{S}_{\mathrm{C}_{i} \in \mathscr{C}}^{\mathscr{C}}\right\},\left\{\boldsymbol{S}_{D_{j} \in \mathscr{D}}^{\mathscr{D}}\right\}\right\}$ is the finite strategy set of different game players. $\boldsymbol{S}^{\mathcal{S}}=\left\{\left(\mathbf{P}_{k}, \mathbb{p}\right) \mid \mathbf{P}_{k} \in\right.$ $\left\{\mathbf{P}_{1} \cdots \mathbf{P}_{M}\right\}$ and $\left.\mathbb{P}=\left[\mathscr{P}_{C_{1}} \cdots \mathscr{P}_{C_{l}}\right]\right\}$ is the strategy combination of the MCP server where $\mathbf{P}_{k}$ is the MCS service price per sensing data bit, and $\mathbb{p}$ is the price distribution vector for DP controllers. For simplicity, $\mathbf{P}_{k}$ is defined as a discrete value, and $\mathbf{P}_{1}, \mathbf{P}_{M}$ are the minimum and maximum prices, respectively, and $\mathscr{P}_{C_{i, 1 \leq i \leq l}}$ is the assigned price per bit for the DP controller $C_{i}$ where $\mathbf{P}_{k}=\sum_{i=1}^{l} \mathscr{P}_{C_{i}}$. Therefore, the MCS price payment $\mathbf{P}$ is shared by multiple DP controllers.

(v) $S_{C_{i} \in \mathscr{C}}^{\mathscr{C}}=\left\{\alpha_{1}^{C_{i}} \cdots \alpha_{M}^{C_{i}}\right\}$ is the strategy set of $C_{i}$; $\alpha$ represents a proportional-split parameter. For simplicity, $\alpha$ is also defined as a discrete value, and $\alpha_{1}, \alpha_{M}$ are the minimum and maximum $\alpha$ values, respectively. Using the $\alpha^{C_{i}}$ value, the $C_{i}$ redistributes the obtained $\mathscr{P}_{C_{i}}$ price to its corresponding mobile devices.

(vi) $S_{D_{j} \in \mathscr{D}}^{\mathscr{D}}=\left\{\left(\mathscr{A}_{k}^{D_{j}}, \varepsilon^{D_{j}}\right) \mid \mathscr{A}_{k}^{D_{j}} \in\left\{\mathscr{A}_{1}^{D_{j}} \cdots \mathscr{A}_{M}^{D_{j}}\right\}, \varepsilon^{D_{j}} \in \mathbb{E}\right\}$ is the strategy combination of the $D_{j}$ where $\mathscr{A}_{k}^{D_{j}}$ is the $D_{j}$ 's sensing data amount for the MCS service and $\varepsilon^{D_{j}}$ is the $D_{j}$ 's DP level. For simplicity, $\mathscr{A}$ is also defined as a discrete value, and $\mathscr{A}_{1}, \mathscr{A}_{M}$ are the minimum and maximum $\mathscr{A}$ values, respectively.

(vii) $\mathbb{U}=\left\{U^{\mathcal{S}},\left\{U_{C_{i} \in \mathscr{C}}^{\mathscr{C}}\right\},\left\{U_{D_{j} \in \mathscr{D}}^{\mathscr{D}}\right\}\right\}$ is the utility function set of game players. $U^{\mathcal{S}}, U_{C_{i}}^{\mathscr{C}}$, and $U_{D_{j}}^{\mathscr{D}}$ represent the utility functions of the MCP server, $C_{i}$ and $D_{j}$, respectively. 
(viii) The $T$ is a time period. The $\mathbb{G}$ is repeated $t \in T<\infty$ time periods with imperfect information.

2.2. Multilevel Game Based MCS Control Algorithms. The MCS server launches sequentially MCS tasks with specific $\mathcal{E}$ LDP levels to multiple mobile devices. Based on his own preference, each mobile device individually decides the amount of sensed data, and its $\varepsilon$-LDP level, that is, $(\mathscr{A}, \varepsilon)$. Simply, we assume that $\varepsilon$ values are decided based on the discrete set $\mathbb{E}=\left\{\varepsilon_{C_{1}}=1.25, \varepsilon_{C_{2}}=1.5, \varepsilon_{C_{3}}=1.75, \varepsilon_{C_{4}}=2\right\}$. Therefore, there are $4 \mathrm{DP}$ controllers; that is, $|\mathbb{E}|=4$, in our MCS platform and each DP controller has its own $\varepsilon$-LDP level. As a mediator, the DP controller actually collects the same $\varepsilon$-LDP level sensed data from some mobile devices.

In this study, we implicitly decompose the MCS scheme into three levels and present dynamic solutions for each level control problem. To achieve a higher payoff, each system agent performs a self-configuration mechanism in order to identify better opportunities. Using the incentive price, the MCS server can induce selfish mobile devices to participate in the MCS process. At the upper level, the MCS server pays the price to DP controllers based on the server's strategies $\mathbf{P}$ and $\mathbf{p}$. At the lower level, each DP controller redistributes the obtained price to its corresponding mobile devices based on the $\alpha$ proportional-split sharing solution. At the same time, mobile devices choose their $\varepsilon$-LDP levels to adaptively respond to the DP controller decision based on the feedback learning process. At the global level, the MCS server and mobile devices interact and adjust their strategies, that is, $\mathbf{P}$ and $\mathscr{A}^{D}$, to maximize individual payoffs. Based on this multilevel game approach, we can reach a fair-efficient solution under dynamically changing system environments.

At the upper level, the MCS server attempts to maximize the service outcome with the lower price payment. However, there is a fundamental tradeoff. If the MCS service price increases, it can induce more selfish devices to participate in MCS services, which will cause a better service outcome while sacrificing the cost effectiveness. Therefore, by considering this tradeoff, the MCS server adaptively selects the most proper price strategy. To capture this conflicting relationship, the MCS server's payoff is defined with the strategy combination $(\mathbf{P}, \mathbb{p})$. In $(\mathbf{P}, \mathbb{p})$, the $\mathbf{P}$ strategy is decided to interact with mobile devices at the global level. Based on the $\mathbf{P}$ strategy, the MCS server adjusts the price vector p for DP controllers in the upper level. To maximize his payoff, the $p$ is decided according to the idea of proportional-split sharing approach. At time $t$, the utility function of MCS server $\left(U_{t}^{\mathcal{S}}\left(\mathbf{P}, \mathbb{p}^{t}\right)\right)$ is defined as follows:

$$
\begin{aligned}
& U_{t}^{\mathcal{S}}\left(\mathbf{P}, \mathbb{P}^{t}=\left[\mathscr{P}_{C_{1}}^{t}, \mathscr{P}_{C_{2}}^{t}, \mathscr{P}_{C_{3}}^{t}, \mathscr{P}_{C_{4}}^{t}\right]\right) \\
&=\sum_{i=1}^{i=4}\left(\Phi_{t}^{C_{i}}\left(\mathscr{P}_{C_{i}}^{t}\right) \times \mathbb{W}\left[\mathscr{P}_{C_{i}}^{t}\right]\right) \\
& \text { s.t., } \quad \mathbb{W}\left[\mathscr{P}_{C_{i}}^{t}\right]\left.\left(1+\frac{\Phi_{t}^{C_{i}}\left(\mathscr{P}_{C_{i}}^{t}\right)}{\max _{C_{j} \in \mathscr{C}} \Phi_{t}^{C_{j}}\left(\mathscr{P}_{C_{j}}^{t}\right)}\right)-\mathscr{P}_{C_{i}}^{t}\right) \\
&=\left(\varepsilon^{C_{i}} \times \log (1)\right. \\
& \text { and } \sum_{i=1}^{i=4} \mathscr{P}_{C_{i}}^{t}=\mathbf{P}
\end{aligned}
$$

where $\varepsilon^{C_{i}}$ is the $\varepsilon$-LDP value for the DP controller $C_{i}$, and $\Phi_{t}^{C_{i}}\left(\mathscr{P}_{C_{i}}^{t}\right)$ is the sensed data amount collected by the $C_{i}$ at time $t$ with the $\mathscr{P}_{C_{i}}^{t}$ payment. For the $t+1$ time period, the $\mathscr{P}_{C_{1}}^{t+1}$ value with the strategy $\mathbf{P}$ is decided based on the $\varepsilon$ proportional-split sharing method:

$$
\mathscr{P}_{C_{i}}^{t+1}(\mathbf{P})=\mathbf{P} \times\left(\frac{\varepsilon^{C_{i}} \times \log \left(1+\Phi_{t}^{C_{i}}\left(\mathscr{P}_{C_{i}}^{t}\right) / \max _{C_{j} \in \mathscr{C}} \Phi_{t}^{C_{j}}\left(\mathscr{P}_{C_{j}}^{t}\right)\right)}{\sum_{C_{k} \in \mathscr{C}}\left(\varepsilon^{C_{k}} \times \log \left(1+\Phi_{t}^{C_{k}}\left(\mathscr{P}_{C_{k}}^{t}\right) / \max _{C_{j} \in \mathscr{C}} \Phi_{t}^{C_{j}}\left(\mathscr{P}_{C_{j}}^{t}\right)\right)\right)}\right)
$$

At the lower level, each DP controller redistributes the obtained price $\mathscr{P}_{C}^{t}$ to its corresponding mobile devices in a distributed manner. According to the participating devices' sensed data amount, the utility function of $C_{i}$ is defined, and the $C_{i}$ selects the strategy $\alpha$ to maximize his utility function; the $\alpha$ value is an element of $S_{C_{i}}^{\mathscr{C}}$, which is a discrete set of real numbers. At time $t$, the utility function of $C_{i}$ with the $\alpha_{x}^{C_{i}, t} \in S_{C_{i}}^{\mathscr{C}}$ strategy $\left(U_{t}^{C_{i}}\left(\alpha_{x}^{C_{i}, t}\right)\right)$ is estimated as follows;

$$
\begin{gathered}
U_{t}^{C_{i}}\left(\alpha_{x}^{C_{i}, t}\right)=\arg \max _{\alpha_{x}^{C_{i}, t} \in S_{C_{i}}^{\mathscr{E}} D_{j} \in \Gamma_{C_{i}}} \sum_{\text {s.t., } \quad}\left(X \times \mathscr{A}^{D_{j}, t}\right) \\
=\left[\alpha_{x}^{C_{i}, t} \times \log \left(1+\frac{\mathscr{A}^{D_{j}, t}}{\max _{D_{k} \in \Gamma_{C_{i}}} \mathscr{A}^{D_{k}, t}}\right)-p_{D_{j}}^{t}\left(\mathscr{A}_{v}^{D_{j}, t}, \mathscr{P}_{C_{i}}^{t}, \alpha_{x}^{C_{i}, t}\right)\right]
\end{gathered}
$$

where $\Gamma_{C_{i}}$ is the set of mobile devices, which are actively participating MCS service through the $C_{i}$. Like the p decision mechanism, the payment mechanism for mobile devices is designed based on the proportional-split sharing approach. 
With the strategy $\alpha$, the $C_{i}$ decides the service price per bit $\left(p_{D_{j}}^{t}(\cdot)\right)$ for the mobile device $D_{j} \in \Gamma_{C_{i}}$. At time $t$, if the $C_{i}$ selects the $\alpha_{1 \leq x \leq M}^{C_{i}, t}$ strategy and the $D_{j}$ contributes to the $\mathscr{A}_{v}^{D_{j}, t}$ amount of sensed data, the $p_{D_{j}}^{t}\left(\mathscr{A}_{v}^{D_{j}, t}, \mathscr{P}_{C_{i}}^{t}, \alpha_{x}^{C_{i}, t}\right)$ is given by

$$
\begin{aligned}
& p_{D_{j}}^{t}\left(\mathscr{A}_{v}^{D_{j}, t}, \mathscr{P}_{C_{i}}^{t}, \alpha_{x}^{C_{i}, t}\right)=\mathscr{P}_{C_{i}}^{t} \\
& \quad \times\left(\frac{\alpha_{x}^{C_{i}, t} \times \log \left(1+\mathscr{A}_{v}^{D_{j}, t} / \max _{D_{k} \in \Gamma_{C_{i}}} \mathscr{A}_{v}^{D_{k}, t}\right)}{\sum_{D_{n} \in \Gamma_{C_{i}}}\left(\alpha_{x}^{C_{i}, t} \times \log \left(1+\mathscr{A}_{v}^{D_{n}, t} / \max _{D_{k} \in \Gamma_{C_{i}}} \mathscr{A}_{v}^{D_{k}, t}\right)\right)}\right)
\end{aligned}
$$

At each time step, the $C_{i}$ receives the payoff as a consequence of selecting strategy. The $C_{i}$ uses this information to predict the payoff values of next time period and adjust the propensities of their strategies. In this study, we adopt the modified Roth-Erev learning mechanism to decide on the $C_{i}$ 's strategy [21]. At time $t+1$, the expected utility of $C_{i}$ with the $\alpha_{x}^{C_{i}, t}$ strategy $\left(\widehat{U}_{t+1}^{C_{i}}\left(\alpha_{x}^{C_{i}}\right)\right)$ is derived as follows:

$$
\widehat{U}_{t+1}^{C_{i}}\left(\alpha_{x}^{C_{i}}\right)= \begin{cases}\widehat{U}_{t}^{C_{i}}\left(\alpha_{x}^{C_{i}}\right)+\left[\Upsilon \times\left(\frac{U_{t}^{C_{i}}-\widehat{U}_{t}^{C_{i}}\left(\alpha_{x}^{C_{i}}\right)}{\max \left(U_{t}^{C_{i}}, \widehat{U}_{t}^{C_{i}}\left(\alpha_{x}^{C_{i}}\right)\right)}\right)\right] & \text { if } \alpha_{x}^{C_{i}} \text { is selected at time } t \\ \widehat{U}_{t}^{C_{i}}\left(\alpha_{x}^{C_{i}}\right), & \text { otherwise }\end{cases}
$$

where $\Upsilon$ is the learning rate and $U_{t}^{C_{i}}$ is the actually obtained utility for the $C_{i}$ at time $t$. Based on this expected utility value, we can calculate the propensity of each strategy $\left(\alpha_{h}^{C_{i}, t} \in S_{C_{i}}^{\mathscr{C}}\right)$ at the $t+1$ time period. Based on the modified Roth-Erev learning mechanism, the propensity of $\alpha_{h}^{C_{i}, t}$ strategy $\left(\zeta_{t+1}^{C_{i}, h}\right)$ at time $t+1$ is defined as [21]:

$$
\begin{aligned}
& \zeta_{t+1}^{C_{i}, h}=(1-\chi) \times \zeta_{t}^{C_{i}, h}+3\left(\epsilon, \widehat{U}_{t}^{C_{i}}\left(\alpha_{h}^{C_{i}}\right), \zeta_{t}^{C_{i}, h}\right) \\
\text { s.t., } & 3\left(\epsilon, \widehat{U}_{t}^{C_{i}}\left(\alpha_{h}^{C_{i}}\right), \zeta_{t}^{C_{i}, h}\right) \\
= & \begin{cases}\frac{\widehat{U}_{t}^{C_{i}}\left(\alpha_{h}^{C_{i}}\right) \times(1-\epsilon)}{\omega}, & \text { if } \alpha_{h}^{C_{i}} \text { is selected at time } t \\
\frac{\widehat{U}_{t}^{C_{i}}\left(\alpha_{h}^{C_{i}}\right) \times \epsilon}{\omega}, & \text { otherwise }\end{cases}
\end{aligned}
$$

where $\omega$ and $\chi$ are the weight and recency parameters, respectively. The parameter $\epsilon$ is used to encourage the exploitation of new actions while avoiding premature convergence on a suboptimal action [21].

At the lower level, individual mobile device selects his $\mathcal{\varepsilon}$ LDP level; it is directly related to decide on his corresponding DP controller. In this study, there are four DP controllers. Therefore, each mobile device has four possible strategies. For the $D_{j}$, his payoff corresponds to the received outcome minus the incurred cost. At time $t$, if the $D_{j}$ selects the $\varepsilon_{C_{i}}^{D_{j}, t}$ strategy for his MCS service, that is, $C_{i}$ is selected as $D_{j}$ 's corresponding DP controller, the payoff of $D_{j}$ with the strategies $\mathscr{A}_{v}^{D_{j}, t}$ and $\varepsilon_{C_{i}}^{D_{j}, t}\left(U_{t}^{D_{j}}\left(\mathscr{A}_{v}^{D_{j}, t}, \varepsilon_{C_{i}}^{D_{j}, t}\right)\right)$ is defined as follows:

$$
\begin{aligned}
U_{t}^{D_{j}} & \left(\mathscr{A}_{v}^{D_{j}, t}, \varepsilon_{C_{i}}^{D_{j}, t}\right) \\
= & \left(\mathscr{A}_{v}^{D_{j}, t} \times p_{D_{j}}^{t}\left(\mathscr{A}_{v}^{D_{j}, t}, \mathscr{P}_{C_{i}}^{t}, \alpha_{h}^{C_{i}, t}\right)\right) \\
& -\left(\beta \times \mathscr{A}_{v}^{D_{j}, t}\right)^{Q}-\left(\omega \times \mathscr{A}_{v}^{D_{j}, t}\right)^{q}, \\
\text { s.t., } \quad Q= & v-\frac{1}{\left(\varepsilon_{C_{i}}^{D_{j}, t}\right)}
\end{aligned}
$$

To estimate the instantaneous expense for MCS services, the cost function is defined based on the sensed data amount $\mathscr{A}_{v}^{D_{j}, t}$ given by $\left(\beta \times \mathscr{A}_{v}^{D_{j}, t}\right)^{\mathrm{Q}}$ and $\left(\omega \times \mathscr{A}_{v}^{D_{j}, t}\right)^{q}$ where $\beta, \omega, q$, and $Q$ are estimation parameters. With the $\mathscr{A}_{v}^{D_{j}, t}$ and $\varepsilon_{C_{i}}^{D_{j}, t}$ strategies, the expected utility of $D_{j}$ at the $t+1$ time period $\left(\widehat{U}_{t+1}^{D_{j}}\left(\mathscr{A}_{v}^{D_{j}, t}, \varepsilon_{C_{i}}^{D_{j}, t}\right)\right)$ is also derived in the same manner as equation (7);

$$
\widehat{U}_{t+1}^{D_{j}}\left(\mathscr{A}_{v}^{D_{j}, t}, \varepsilon_{C_{i}}^{D_{j}, t}\right)= \begin{cases}\widehat{U}_{t}^{D_{j}}\left(\mathscr{A}_{v}^{D_{j}, t}, \varepsilon_{C_{i}}^{D_{j}, t}\right)+\left[\kappa \times\left(\frac{U_{t}^{D_{j}}-\widehat{U}_{t}^{D_{j}}\left(\mathscr{A}_{v}^{D_{j}, t}, \varepsilon_{C_{i}}^{D_{j}, t}\right)}{\max \left(U_{t}^{D_{j}}, \widehat{U}_{t}^{D_{j}}\left(\mathscr{A}_{v}^{D_{j}, t}, \varepsilon_{C_{i}}^{D_{j}, t}\right)\right)}\right)\right] & \text { if } \varepsilon_{C_{i}, t}^{D_{j}, t} \text { is selected at time } t \\ \widehat{U}_{t}^{D_{j}}\left(\mathscr{A}_{v}^{D_{j}, t}, \varepsilon_{C_{i}}^{D_{j}, t}\right) & \text { otherwise }\end{cases}
$$

where $\kappa$ is the learning rate and $U_{t}^{D_{j}}$ is the actually obtained utility for the $D_{j}$ at time $t$. Based on this expected utility value, we also calculate the propensity of strategy $\varepsilon_{C_{1 \leq i \leq 4}}^{D_{j}, t} \in \mathbb{E}$ at time $+1\left(\zeta_{t+1}^{D_{j}, i}\right)$. It is also estimated based on the modified Roth-Erev learning mechanism. Therefore, the $\zeta_{t+1}^{D_{j}, i}$ value is calculated in the same manner according to (6). 
Based on the strategy propensities, we can estimate the probability distribution for each strategy selection. In the proposed algorithms, the softmax activation logistic function is adopted to calculate the probability distribution [22]. Finally, at the lower level, the $C_{i}$ and $D_{j}$ select their strategies, that is, $\alpha^{C_{i}}$ and $\varepsilon^{D_{j}}$, according to these distributions. Therefore, without any impractical rationality assumptions, players dynamically change their current strategies while interacting with other players and can learn how to perform well in an effort to maximize their own goals.
At the global level, the MCS server and individual mobile devices dynamically adjust their strategies, that is, $\mathbf{P}$ and $\mathscr{A}$, to maximize their payoffs. To decide on $\mathbf{P}$ and $\mathscr{A}$ strategies, the time period of the global level $(\Delta T)$ is different from the time period $(\Delta t)$ of the upper or lower level process; $\Delta T$ is larger than $\Delta t$ where $\Delta t \ll \Delta T$. From the viewpoint of the MCS server, the expected payoff with the strategy $\mathbf{P}_{1 \leq j \leq M}^{T}\left(\widehat{U}_{T+1}^{\mathcal{S}}(\cdot)\right)$ at the $T+1$ time period can be defined as

$$
\widehat{U}_{T+1}^{\mathcal{S}}\left(\mathbf{P}_{j}^{T}, \mathbb{p}^{(\cdot)}\right)= \begin{cases}\widehat{U}_{T}^{\mathcal{S}}\left(\mathbf{P}_{j}^{T}, \mathbb{p}^{(\cdot)}\right)+\left[\partial \times\left(\frac{U_{T}^{\mathcal{S}}-\widehat{U}_{T}^{\mathcal{S}}\left(\mathbf{P}_{j}^{T}, \mathbb{p}^{(\cdot)}\right)}{\max \left(U_{T}^{\mathcal{S}}, \widehat{U}_{T}^{\mathcal{S}}\left(\mathbf{P}_{j}^{T}, \mathbb{p}^{(\cdot)}\right)\right)}\right)\right] & \text { if } \mathbf{P}_{j}^{T} \text { is selected at time } T \\ \widehat{U}_{T}^{\mathcal{S}}\left(\mathbf{P}_{j}^{T}, \mathbb{p}^{(\cdot)}\right), & \text { otherwise }\end{cases}
$$

$$
\text { s.t., } \quad U_{T}^{\mathcal{S}}=\sum_{t \in \Delta T} U_{t}^{\mathcal{S}}\left(\mathbf{P}, p^{t}\right)
$$

where $\partial$ is the learning rate and $\mathbf{P}_{j}^{T}$ is the $j^{\text {th }}$ strategy in $\left\{\mathbf{P}_{1} \cdots \mathbf{P}_{M}\right\} . \mathbb{p}^{(\cdot)}$ is the set of multiple $\mathscr{P}_{C}$ strategies, which are selected during the $\Delta T$. According to the $\widehat{U}_{T+1}^{\mathcal{S}}$ value, we can also calculate the propensity of $\mathbf{P}_{j}$ strategy $\left(\Psi_{T+1}^{\mathcal{S}, j}\right)$ at the $T+1$ time period. In this case, $\Psi_{T+1}^{\mathcal{S}, j}$ is estimated as follows:

$$
\begin{aligned}
\Psi_{T+1}^{\mathcal{S}, j}=\Psi_{T}^{\mathcal{S}, j}+ & {\left[\mu \times\left(\frac{U_{T}^{\mathcal{S}}-\widehat{U}_{T}^{\mathcal{S}}\left(\mathbf{P}_{j}^{T}, \mathbb{p}^{(\cdot)}\right)}{\max \left(U_{T}^{\mathcal{S}}, \widehat{U}_{T}^{\mathcal{S}}\left(\mathbf{P}_{j}^{T}, \mathbb{p}^{(\cdot)}\right)\right)}\right) \times \mathscr{H}\left(\Psi_{T}^{\mathcal{S}, j}, \sigma_{T}^{j}\right)\right] } \\
\text { s.t., } \quad \mathscr{H}\left(\Psi_{T}^{\mathcal{S}, j}, \sigma_{T}^{j}\right) & =\frac{\phi\left(\sigma_{T}^{j}\right)}{\left(1+\exp \left(-\Psi_{T}^{\mathcal{S}, j} / \tau\right)\right)} \\
\text { and } \phi\left(\sigma_{T}^{j}\right) & = \begin{cases}1, & \text { if } \sigma_{T}^{1 \leq j \leq M}=1 \\
-1, & \text { otherwise }\end{cases}
\end{aligned}
$$

where $\mu$ is the learning rate, and $\tau$ is the control factor. $\mathscr{H}(\cdot)$ is the gradient of the sigmoid function with respect to the $\Psi$. If the $\mathbf{P}_{j}$ strategy is selected at the $T$ time period, $\sigma_{T}^{j}$ is set to 1 , that is, $\sigma_{T}^{j}=1$; otherwise $\sigma_{T}^{j}$ is set to 0 where $\sigma_{T}^{j}=\{0,1\}$ [21].

With the interaction with the MCS server, each individual mobile device dynamically adjusts his strategy $\mathscr{A}$ to maximize the payoff. According to (7), the $D_{j}$ 's payoff with the strategies $\mathscr{A}_{v}^{D_{j}, T}$ and $\varepsilon_{(\cdot)}^{D_{j}, T}$ is defined as follows:

$$
\begin{array}{ll} 
& U_{T}^{D_{j}}\left(\mathscr{A}_{v}^{D_{j}, T}, \varepsilon_{(\cdot)}^{D_{j}, T}\right)=\mathrm{I}_{T}^{D_{j}}\left(\mathscr{A}_{v}^{D_{j}, T}\right)-c\left(\mathscr{A}_{v}^{D_{j}, T}, \varepsilon_{(\cdot)}^{D_{j}, T}\right) \\
\text { s.t., } \quad & \left\{\begin{array}{l}
\mathrm{I}_{T}^{D_{j}}\left(\mathscr{A}_{v}^{D_{j}, T}, \varepsilon_{(\cdot)}^{D_{j}, T}\right)=\sum_{t \in \Delta T} p_{D_{j}}^{t}\left(\mathscr{A}_{v}^{D_{j}, t}, \mathscr{P}_{C_{i}}^{t}, \alpha_{h}^{C_{i}, t}\right) \\
c\left(\mathscr{A}_{v}^{D_{j}, T}\right)=\sum_{t \in \Delta T}\left(\left(\beta \times \mathscr{A}_{v}^{D_{j}, t}\right)^{Q}+\left(\omega \times \mathscr{A}_{v}^{D_{j}, t}\right)^{q}\right)
\end{array}\right.
\end{array}
$$

where $\varepsilon_{(\cdot)}^{D_{j}, T}$ is the set of multiple $\varepsilon^{D_{j}}$ strategies, which are selected during the $\Delta T$. With the $\mathscr{A}_{v}^{D_{j}, T}$ and $\varepsilon_{(\cdot)}^{D_{j}, T}$ strategies, the expected payoff of $D_{j}$ at the $T+1$ time period $\left(\widehat{U}_{T+1}^{D_{j}}\left(\mathscr{A}_{v}^{D_{j}, T}, \mathcal{\varepsilon}_{(\cdot)}^{D_{j}, T}\right)\right)$ is also expected according to (9). Based on this information, we also calculate the propensity of strategy $\mathscr{A}_{1 \leq k \leq M}^{D_{j}, T}$ at time $T+1\left(\eta_{t+1}^{D_{j}, k}\right) ; \eta_{t+1}^{D_{j}, k}$ is also calculated in the same manner as equation (10). Based on these strategy propensities, that is, $\Psi$ and $\eta$, we also estimate the probability distributions for $\mathbf{P}$ and $\mathscr{A}$ strategies. In the same manner as in the lower level game process, we use the softmax activation logistic function to calculate these probability distributions. According to these distributions, the MCS server and each mobile device select their strategies, that is, $\mathbf{P}$ and $\mathscr{A}^{D}$. In each time round $\Delta T$, game players can learn the others' responses with incoming information and will select better strategies to approximate the most desirable system solution. 
TABLE 1: System parameters used in the simulation experiments.

\begin{tabular}{|c|c|c|c|}
\hline Sensing Tasks & Total MCS cycles & $\varepsilon$-LDP value & Service Duration \\
\hline $\mathbb{S}_{1}$ & $4.5 \mathrm{~K}$ sensing cycles $/ \mathrm{s}$ & 0.1 & $3,000 \mathrm{sec}(50 \mathrm{~min})$ \\
\hline $\mathbb{S}_{2}$ & $6.5 \mathrm{~K}$ sensing cycles/s & 0.15 & $2,700 \mathrm{sec}(45 \mathrm{~min})$ \\
\hline $\mathbb{S}_{3}$ & $9 \mathrm{~K}$ sensing cycles/s & 0.2 & $2,880 \mathrm{sec}(48 \mathrm{~min})$ \\
\hline $\mathbb{S}_{4}$ & $12 \mathrm{~K}$ sensing cycles/s & 0.25 & $2,760 \mathrm{sec}(46 \mathrm{~min})$ \\
\hline Parameter & Value & \multicolumn{2}{|c|}{ Description } \\
\hline$l$ & 4 & \multicolumn{2}{|c|}{ the number of DP controllers } \\
\hline$n$ & 100 & \multicolumn{2}{|c|}{ the number of mobile devices } \\
\hline$\varepsilon_{1}, \varepsilon_{M}$ & $1.25,2$ & \multicolumn{2}{|c|}{ the minimum and maximum privacy levels } \\
\hline $\mathbf{P}_{1}, \mathbf{P}_{2}, \mathbf{P}_{3}, \mathbf{P}_{4}, \mathbf{P}_{M=5}$ & $1,1.5,2,2.5,3$ & \multicolumn{2}{|c|}{ the MCS service price per bit } \\
\hline $\mathscr{A}_{1}, \mathscr{A}_{M=5}$ & $200,600 \mathrm{cycles} / \mathrm{s}$ & \multicolumn{2}{|c|}{ the minimum and maximum sensing data amounts } \\
\hline$\alpha_{1}, \alpha_{M=4}$ & $1.2,2$ & \multicolumn{2}{|c|}{ the minimum and maximum proportional sharing factors } \\
\hline$\Upsilon$ & 0.1 & \multicolumn{2}{|c|}{ the learning rate for DP controllers } \\
\hline$\chi$ & 0.9 & \multicolumn{2}{|c|}{ the recency parameter to select a DP controller } \\
\hline$\epsilon$ & 0.5 & \multicolumn{2}{|c|}{ the parameter to avoid a premature convergence } \\
\hline$\omega$ & 4 & \multicolumn{2}{|c|}{ the weight parameter to select a DP controller } \\
\hline$\beta, \nu$ & $1.5,1$ & \multicolumn{2}{|c|}{ the estimation parameters to calculate the DP cost } \\
\hline$\omega, q$ & $1.1,0.7$ & \multicolumn{2}{|c|}{ the estimation parameters to calculate the service cost } \\
\hline$\kappa$ & 1.5 & \multicolumn{2}{|c|}{ the learning rate for mobile devices } \\
\hline$\partial, \mu$ & $0.1,0.1$ & \multicolumn{2}{|c|}{ the learning rate parameters for the MCS servers } \\
\hline$\tau$ & 0.5 & \multicolumn{2}{|c|}{ the control factor for the MCS server } \\
\hline
\end{tabular}

2.3. Main Steps of Proposed Multilevel MCS Control Scheme. In this study, we have investigated the influence of crowdsensing information with the privacy protection issue and propose a novel MCS control scheme based on the multilevel interactive game model. It takes into account the desired level of privacy protection, computational efficiency, individual rationality, and social welfare. As game players, the MCS server, DP controllers, and mobile devices can capture the dynamics of MCS system conditions and determine their best strategies to maximize their payoffs. Through a step-by-step online learning approach, they are able to feed back their decisions while getting a full synergy of interactive multilevel operations. Therefore, game players at each level adaptively adjust their control decisions individually without centralized coordination. It is an effective and suitable way to operate practically the real-world MCS system. The main steps of the proposed MCS control scheme are described as follows.

Step 1. At the initial time, system parameters and sensing tasks are characterized by the simulation scenario and Table 1.

Step 2. Each DP controller has been designated as a specific $\mathcal{E}$ LDP level. Therefore, the collected data in each DP controller have the same $\varepsilon$-LDP value.

Step 3. The MCS generates sequentially MCS sensing tasks, and mobile devices actively sense and collect the information of local environments. As mediators, DP controllers arbitrate between the MCS server and mobile devices.

Step 4. The MCS server selects the combination of strategies $(\mathbf{P}, \mathbf{p})$. At the global level, the MCS server decides on the $\mathbf{P}$ value at each $\Delta T$ according to (9)-(10). At the upper level, the MCS server dynamically adjusts the price vector $p$ for DP controllers at each $\Delta t$ according to (2).

Step 5. DP controllers redistribute the obtained price $\mathscr{P}_{C}$ to their corresponding mobile devices in the $\alpha$ proportionalsplit sharing approach. At the lower level, each DP controller dynamically selects his strategy $\alpha^{C}$ at each $\Delta t$ to maximize the $U^{C}$ according to (3), (5)-(6).

Step 6. Individual mobile devices select the combination of their strategies $(\mathscr{A}, \varepsilon)$. At the global level, they dynamically adjust their strategies $\mathscr{A}$ at each $\Delta T$ using (11). At the lower level, they decide on their $\varepsilon$-LDP values at each $\Delta t$ according to (7)-(8).

Step 7. Based on the step-by-step multilevel game process, the MCS, DP controllers and mobile devices interact with one another and cause a cascade effect in an online distributed fashion.

Step 8. By using the proportional-split sharing and online learning methods, game players know how to select their strategies while maximizing their perceived payoffs.

Step 9. Under the dynamic MCS system environment, game players are constantly self-monitoring the current MCS system conditions; proceed to Step 3 for the next multilevel game iteration.

\section{Performance Evaluation}

In this section, we evaluate the performance of our proposed protocol and compare it with that of the existing PPMC [16] 
and SDLP [19] schemes. Based on the simulation results, we confirm the superiority of the proposed approach. To ensure a fair comparison, the following simulation assumptions and MCS system platform are used.

(i) There are one MCS server $\mathcal{S}$, four DP controllers $\mathscr{C}=$ $\left\{C_{1}, C_{2}, C_{3}, C_{4}\right\}$ for different DP services, and one hundred mobile devices $\mathscr{D}=\left\{D_{1}, \ldots, D_{100}\right\}$, which are distributed randomly in a geographical region.

(ii) There is the set of MCS sensing application tasks where $\mathbb{A}=\left\{A_{1} \cdots A_{v}\right\}$. A can be categorized as four different sensing task groups, that is, $\left\{\mathbb{S}_{1}, \mathbb{S}_{2}, \mathbb{S}_{3}, \mathbb{S}_{4}\right\}$. Each $\mathbb{S}$ is specified according to the sensing requirements. They are generated with equal probability.

(iii) To distribute the obtained price to its corresponding mobile devices, the strategy set of DP controller is $S^{\mathscr{C}}=\left\{\alpha_{1}^{C}=1.2, \alpha_{2}^{C}=1.4, \alpha_{3}^{C}=1.75, \alpha_{M=4}^{C}=2\right\}$.

(iv) The $\varepsilon$-LDP value of each sensing task application is randomly decided among $\mathbb{E}=\left\{\varepsilon_{C_{1}}=1.25, \varepsilon_{C_{2}}=\right.$ $\left.1.5, \varepsilon_{C_{3}}=1.75, \varepsilon_{C_{4}}=2\right\}$.

(v) Each mobile device has a range of sensing capacity $\mathscr{A}$ to proceed MCS services; $\mathscr{A}$ is a discrete value where $\mathscr{A} \in\left\{\mathscr{A}_{1}=200\right.$ sensing cycles/s, $\mathscr{A}_{2}=300$ sensing cycles/s, $\mathscr{A}_{3}=400$ sensing cycles/s, $\mathscr{A}_{4}=500$ sensing cycles/s, $\mathscr{A}_{M=5}=600$ sensing cycles/s\}.

(vi) Sensing tasks in $\mathbb{A}$ are generated based on the Poisson process; the generation rate range $\lambda$ is varied from 0 to 3 .

(vii) The time period of the global level $(\Delta T)$ is divided into 10 time periods of the upper and lower level process $(\Delta t)$. In this simulation model, $\Delta t$ is assumed as one second.

(viii) System performance measures obtained on the basis of 100 simulation runs are plotted as functions of the sensing task generation rate.

(ix) For simplicity, we assume the absence of physical obstacles in the experiments.

To demonstrate the validity of our proposed method, we measured the sensing task success ratio, MCS participating ratio, and normalized payoff of participating devices. Table 1 shows the system parameters used in the simulation. Major system control parameters of the simulation, presented in Table 1, facilitate the development and implementation of our simulator.

Figure 2 gives the performance comparison of each scheme in terms of the task success ratio. From the viewpoint of system operator, it is a main concern and important performance criterion. As mentioned earlier, the MCS server generates sequentially MCS sensing tasks. As the task generation rate increases, the task success ratio decreases. From Figure 2, it is easy to see that the scheme which is designed in this paper has the best performance. In the proposed scheme, the MCS server, DP controllers, and mobile devices make intelligent control decisions while working together toward an appropriate system performance. Therefore, they can respond practically to current system conditions and

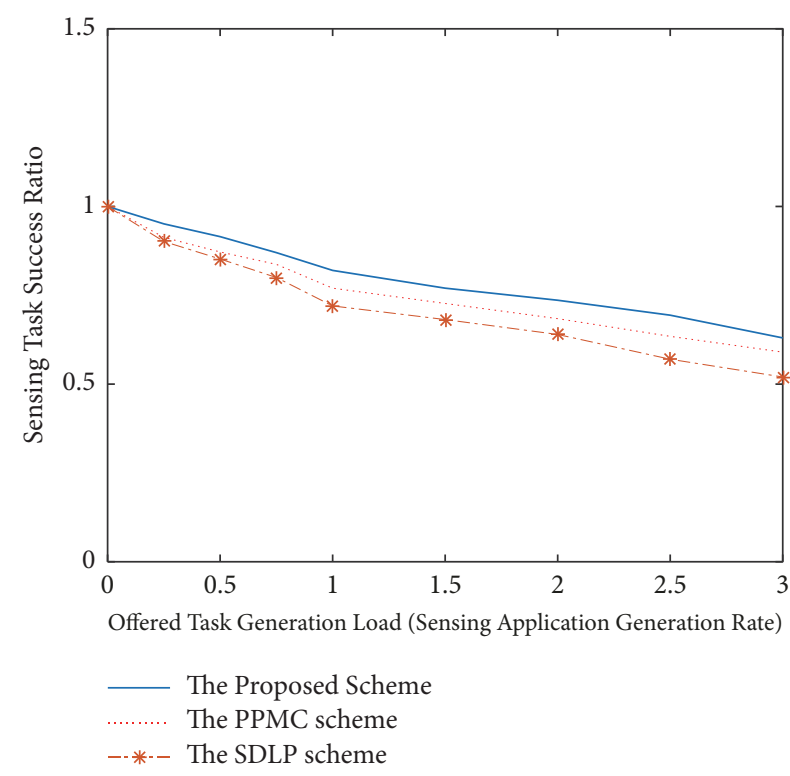

FIGURE 2: Sensing task success ratio.

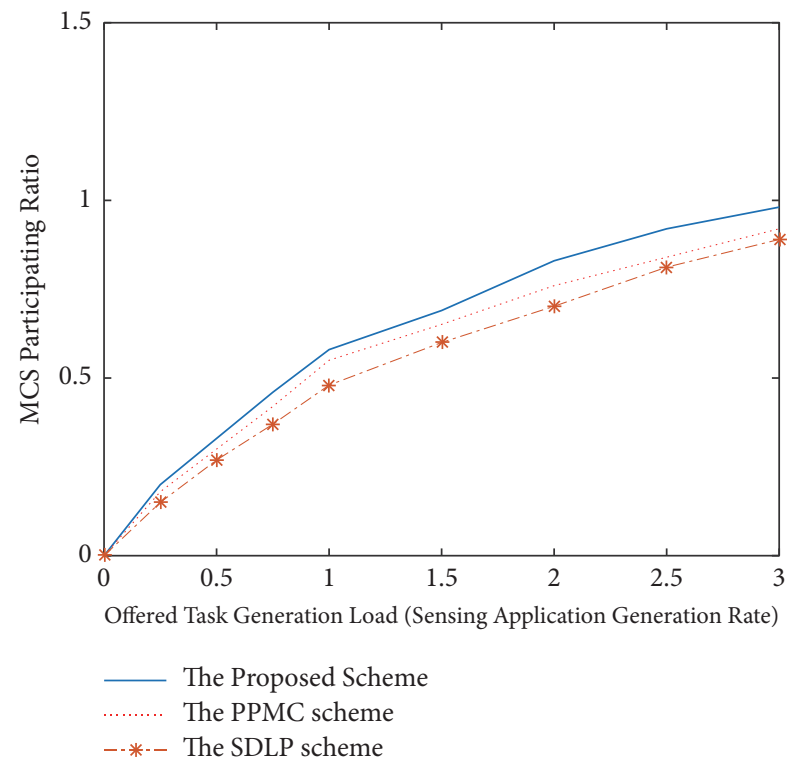

FIGURE 3: MCS participating ratio.

jointly reach a mutually acceptable agreement to complete the request tasks; it leads to a higher task success ratio. From low to high task generation rate, we can effectively complete the requested task sensing applications compared with other existing schemes.

Figure 3 presents the MCS participating ratio for each scheme. In the proposed approach, the MCS server and DP controllers can effectively induce selfish mobile devices to participate in the MCS process. Based on the feedback based online learning methods, the MCS server chooses the most adaptable service price and DP controllers rationally redistribute the assigned price to mobile devices. This multilevel approach can stimulate mobile devices to participate 


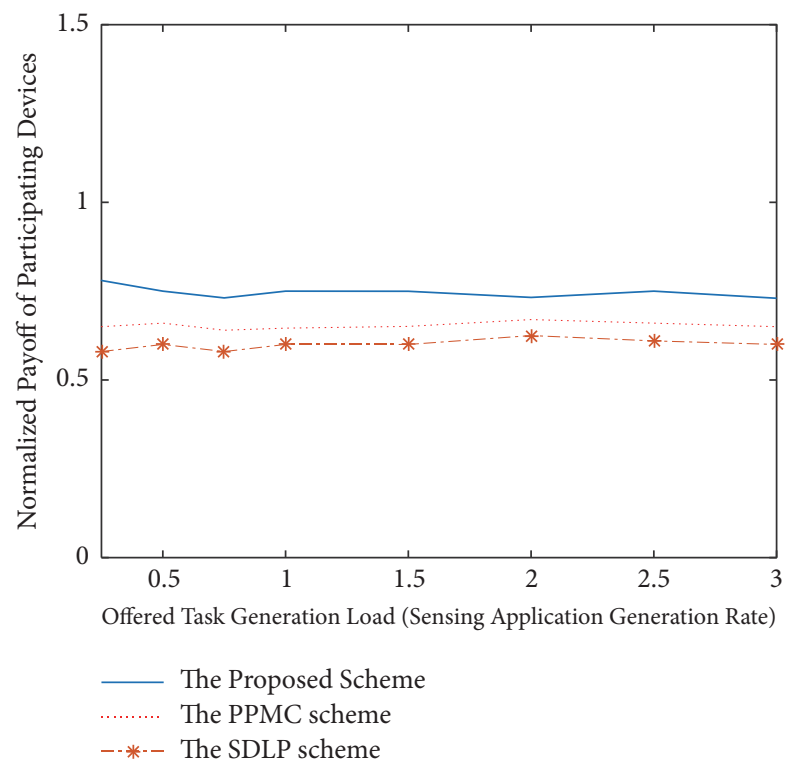

FIGURE 4: Normalized payoff of participating devices.

actively in the MCS process while revealing their private propensities and to take appropriate actions. As game players, the MCS server, DP controllers, and mobile devices are mutually dependent and monitoring each other in order to obtain the best solution for all. For this reason, the proposed scheme can attain the better MCS participating ratio to the existing PPMC and SDLP schemes.

The curves in Figure 4 indicate the normalized payoff of participating mobile devices. In our scheme, mobile devices can get payoffs according to the actual contributions for MCS services. In particular, individual devices in the proposed scheme dynamically select their strategies while aligning their goals and preferences. Therefore, they can maximize their payoffs in a self-adapting manner to meet their goals under dynamically changeable MCS environments. During different task generation intensities, we can see that the proposed scheme achieves better devices' payoffs. In summary, simulation results shown in Figures 2-4 demonstrate that the proposed scheme, which uses a multilevel game model, can get the full synergy of MCS processing operations. In conclusion, simulation results show that our scheme attains an attractive MCS system performance, something that the $P P M C$ [16] and SDLP [19] schemes cannot offer.

\section{Summary and Conclusions}

In recent years, the widespread prevalence of smart devices has enabled a new IoT paradigm, called MCS services. This technique leverages pervasive mobile devices to efficiently collect the big sensory data while rewarding their work. However, they concern about the loss of individual privacy seriously. In this paper, we design a privacy-preserving MCS control scheme based on the multilevel game model. As game players, the MCS server, DP controllers, and mobile devices aim to maximize their payoffs in each level. From the perspectives of the MCS server, DP controllers, and mobile devices, the main concerns are to decide on the service price, to redistribute the obtained price, and to decide on the MCS contribution considering the privacy preservation, respectively. To capture the dynamics of interactive relationships, each game player learns how to perform well by interacting with other players without any impractical rationality assumptions. Using the step-by-step repeated game process, the proposed scheme can explore an effective solution under widely different and diversified MCS situations. It is a practical and suitable approach in the real-world system operation. Through extensive simulation experiments, we evaluate the performance of our scheme and existing protocols, and the results validate that our proposed scheme can significantly improve performance. In the future, we will extend the proposed approach by adding the cooperative game concept as a negotiation bargaining solution. Also, we plan to focus on implementing a realistic MCS application for traffic information collection against faked sensing attacks. Finally, we would like to take a research opportunity to address the big data mining and deep learning issues in the MCS system from the operator's perspective.

\section{Data Availability}

The data used to support the findings of this study are available from the corresponding author upon request.

\section{Conflicts of Interest}

The authors declare that they have no conflicts of interest.

\section{Acknowledgments}

This research was supported by the MSIT (Ministry of Science and ICT), Korea, under the ITRC (Information Technology Research Center) support program (IITP-2018-20180-01799) supervised by the IITP (Institute for Information \& communications Technology Promotion).

\section{References}

[1] J. Ni, K. Zhang, X. Lin, and X. S. Shen, "Securing Fog Computing for Internet of Things Applications: Challenges and Solutions," IEEE Communications Surveys \& Tutorials, vol. 20, no. 1, pp. 601-628, 2018.

[2] K. Kim, S. Uno, and M. Kim, "Adaptive QoS mechanism for wireless mobile network," Journal of Computing Science and Engineering, vol. 4, no. 2, pp. 153-172, 2010.

[3] I. Jang, D. Pyeon, S. Kim, and H. Yoon, "A survey on communication protocols for wireless sensor networks," Journal of Computing Science and Engineering, vol. 7, no. 4, pp. 231-241, 2013.

[4] B. Oh, Y. Na, J. Yang, S. Park, J. Nang, and J. Kim, "Genetic algorithm-based dynamic vehicle route search using car-tocar communication," Advances in Electrical and Computer Engineering, vol. 10, no. 4, pp. 81-86, 2010.

[5] A. Jian, G. Xiaolin, Y. Jianwei, S. Yu, and H. Xin, "Mobile Crowd Sensing for Internet of Things: A Credible Crowdsourcing Model in Mobile-Sense Service," in Proceedings of the 1st IEEE 
International Conference on Multimedia Big Data, BigMM 2015, pp. 92-99, China, April 2015.

[6] L. Xiao, T. Chen, C. Xie, H. Dai, and H. V. Poor, "Mobile Crowdsensing Games in Vehicular Networks," IEEE Transactions on Vehicular Technology, vol. 67, no. 2, pp. 1535-1545, 2018.

[7] Sungwook Kim, Game Theory Applications in Network Design, IGI Global, Hershey, Pa, USA, 2014.

[8] J. Liu, H. Shen, and X. Zhang, "A survey of mobile crowdsensing techniques: A critical component for the internet of things," in Proceedings of the 25th International Conference on Computer Communications and Networks, ICCCN 2016, 6, 1 pages, USA, August 2016.

[9] Aris Gkoulalas-Divanis, Kun Liu, Vassilios S. Verykios, and Ran Wolff, "Preface for the Special Issue on Privacy-Aspects of Data Mining," JCSE, vol. 5, no. 3, pp. 167-168, 2011.

[10] Y. Liu, Y. L. Sun, J. Ryoo, S. Rizvi, and A. V. Vasilakos, "A survey of security and privacy challenges in cloud computing: solutions and future directions," Journal of Computing Science and Engineering, vol. 9, no. 3, pp. 119-133, 2015.

[11] T. Kang, X. Li, C. Yu, and J. Kim, "A Survey of Security Mechanisms with Direct Sequence Spread Spectrum Signals," Journal of Computing Science and Engineering, vol. 7, no. 3, pp. 187-197, 2013.

[12] H. H. Nguyen, J. Kim, and Y. Kim, "Differential privacy in practice," Journal of Computing Science and Engineering, vol. 7, no. 3, pp. 177-186, 2013.

[13] C. Dwork, "Differential privacy," in Automata, Languages and Programming, vol. 4052 of Lecture Notes in Computer Science, pp. 1-12, Springer, Berlin, Germany, 2006.

[14] J. W. Kim, D.-H. Kim, and B. Jang, "Application of Local Differential Privacy to Collection of Indoor Positioning Data," IEEE Access, vol. 6, pp. 4276-4286, 2018.

[15] J. Chen, H. Ma, D. Zhao, and L. Liu, "Correlated Differential Privacy Protection for Mobile Crowdsensing," IEEE Transactions on Big Data, 2018.

[16] J. Lin, D. Yang, M. Li, J. Xu, and G. Xue, "Frameworks for Privacy-Preserving Mobile Crowdsensing Incentive Mechanisms," IEEE Transactions on Mobile Computing, 2018.

[17] M. Huai, L. Huang, Y.-E. Sun, and W. Yang, "Efficient PrivacyPreserving Aggregation for Mobile Crowdsensing," in Proceedings of the 5th IEEE International Conference on Big Data and Cloud Computing, BDCloud 2015, pp. 275-280, China, August 2015.

[18] Y. Sei and A. Ohsuga, "Differential Private Data Collection and Analysis Based on Randomized Multiple Dummies for Untrusted Mobile Crowdsensing," IEEE Transactions on Information Forensics and Security, vol. 12, no. 4, pp. 926-939, 2017.

[19] L. Wang, D. Zhang, D. Yang, B. Y. Lim, and X. Ma, "Differential location privacy for sparse mobile crowdsensing," in Proceedings of the 16th IEEE International Conference on Data Mining, ICDM 2016, pp. 1257-1262, Spain, December 2016.

[20] H. Shin, S. Kim, J. Shin, and X. Xiao, "Privacy Enhanced Matrix Factorization for Recommendation with Local Differential Privacy," IEEE Transactions on Knowledge and Data Engineering, 2018.

[21] G. Alnwaimi, S. Vahid, and K. Moessner, "Dynamic heterogeneous learning games for opportunistic access in LTE-based macro/femtocell deployments," IEEE Transactions on Wireless Communications, vol. 14, no. 4, pp. 2294-2308, 2015.

[22] P. Reverdy and N. E. Leonard, "Parameter estimation in softmax decision-making models with linear objective functions," IEEE
Transactions on Automation Science and Engineering, vol. 13, no. 1, pp. 54-67, 2016. 


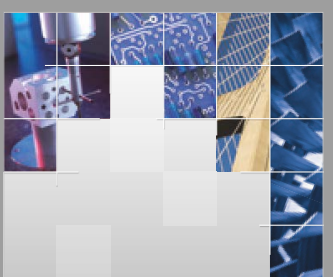

\section{Enfincering}
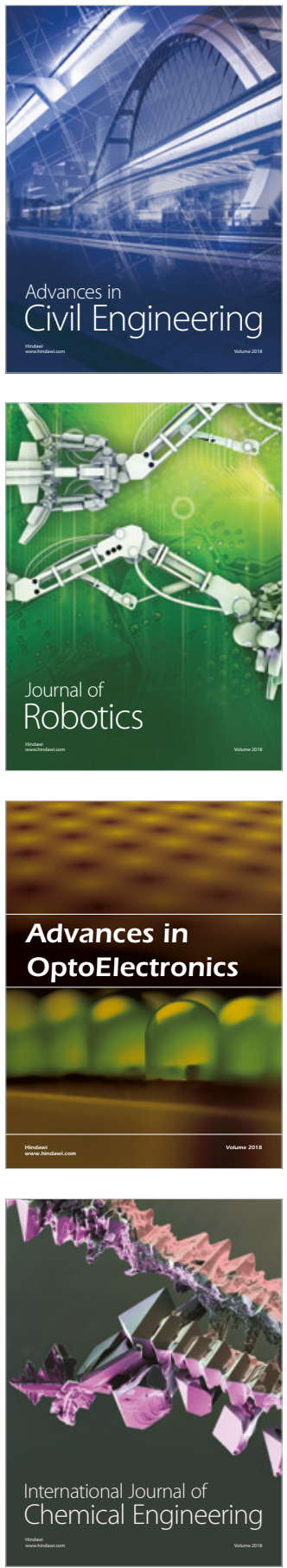

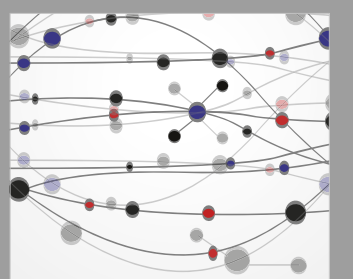

\section{Rotating \\ Machinery}

The Scientific World Journal

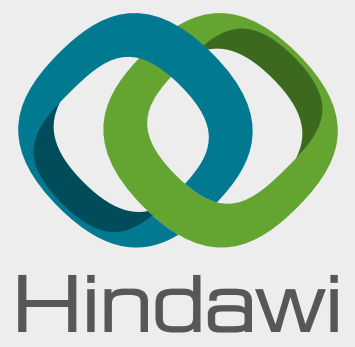

Submit your manuscripts at

www.hindawi.com
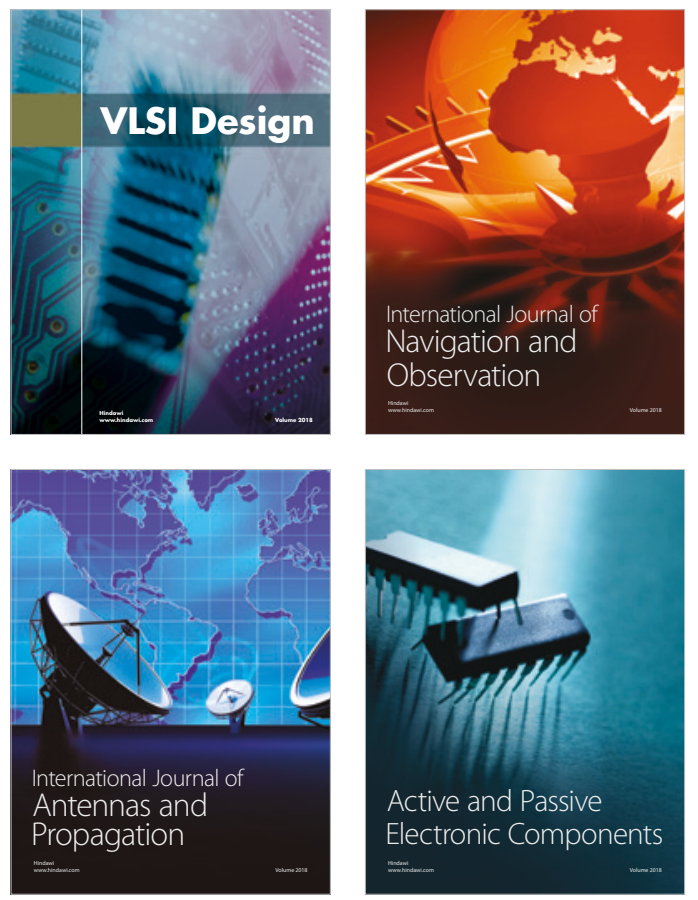
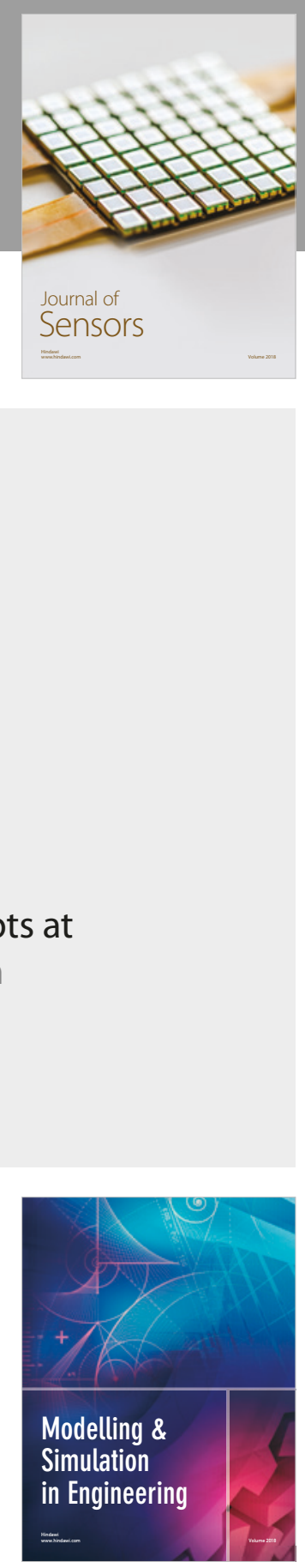

\section{Advances \\ Multimedia}
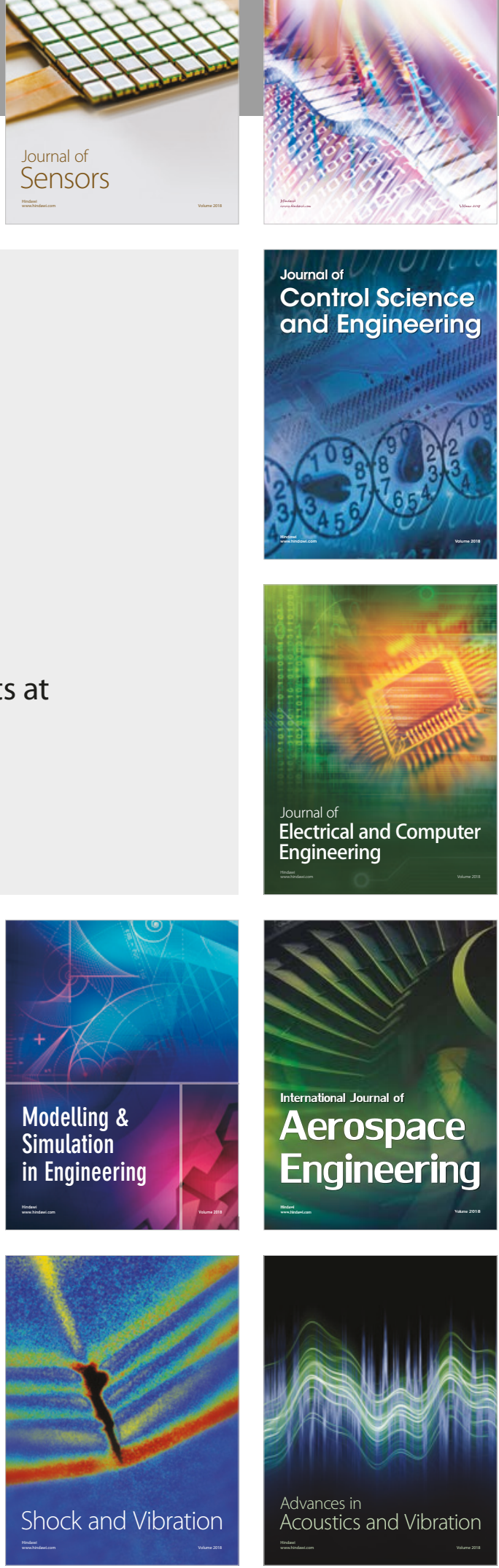